\title{
ORIGINAL
}

\section{CARACTERÍSTICAS CLÍNICAS Y PSICOSOCIALES DE PERSONAS \\ CON FIBROMIALGIA. REPERCUSIÓN DEL DIAGNÓSTICO SOBRE SUS ACTIVIDADES (*)} \author{
Olry de Labry Lima (2) y Juncal Plazaola Castaño (2) \\ (1) Servicio de Medicina Preventiva. Hospital Universitario Virgen de las Nieves. Granada. \\ (2) Area de Investigación. Escuela Andaluza de Salud Pública. Granada
}

María del Carmen Ubago Linares (1), Isabel Ruiz Pérez (2), María José Bermejo Pérez (1), Antonio

\section{RESUMEN}

Fundamento: Actualmente se sabe muy poco de las características de las personas diagnosticadas de fibromialgia, su grado de incapacidad y la respuesta del sistema sanitario. Los objetivos de este trabajo son: conocer el perfil sociodemográfico, clínico y psicosocial de las y los pacientes con fibromialgia; describir la respuesta que obtienen del sistema sanitario, y estudiar la repercusión de este síndrome en las actividades habituales de las personas que lo padecen, incluyendo las del ámbito laboral.

Métodos: Estudio descriptivo transversal. La información se recogió mediante encuesta telefónica realizada a personas diagnosticadas de fibromialgia en el Hospital Universitario Virgen de las Nieves de Granada durante el año 2003.

Resultados: El 92,1\% de las personas entrevistadas eran mujeres. La fibromialgia causó baja laboral en el 31,4\% de los casos. El $64 \%$ percibía su salud como mala o muy mala. La fibromialgia se asoció a otras enfermedades en un 52,3\% de los casos. El médico de familia es el especialista consultado antes del diagnóstico en el $92,1 \%$. El 50,5\% tenía antecedentes de trastorno psiquiátrico, presentándolo en el momento de la entrevista el 36,4\%. El 71\% de la muestra recibía apoyo familiar, señalando un fuerte impacto del síndrome en su entorno un $70,1 \%$ de los casos.

Conclusiones: La fibromialgia fue diagnosticada mayoritariamente en mujeres. Causa una mala percepción del estado de salud y situaciones de incapacidad laboral, afectando negativamente al entorno familiar de la persona que la padece.

Palabras clave: Fibromialgia. Factores psicosociales. Estado de salud. Sistema sanitario.

Correspondencia:

Isabel Ruiz Pérez

Escuela Andaluza de Salud Pública

Campus Universitario de Cartuja.

Apartado de correos 2070

18080 Granada.

Correo electrónico: isabel.ruiz.easp@juntadeandalucia.es
ABSTRACT

Clinical and Psychosocial Characteristics of Subjects with Fbromyalgia. Impact of the Diagnosis on Patients' Activities

Background: Little is known today about the characteristics of ndividuals diagnosed with fibromyalgia, their degree of disability and the health system response system response to this condition. The objectives of this work include: to establish the sociodemographic, clinical and psychosocial profile of male and female patients with fibromyalgia. To describe the response they receive from the health service, and to study the repercussions of this syndrome on the daily activities of subjects who suffer it, including its effects on their work environment.

Methods: Descriptive cross-sectional study. The information was received from telephone interviews of individuals diagnosed with fibromyalgia in the Hospital Universitario Virgen de las Nieves in Granada during 2003.

Results: $92.1 \%$ of those interviewed were women. Fibromyalgia caused work absenteeism in $31.4 \%$ of cases; and $64 \%$ regarded their health as poor or very poor. Fibromyalgia was associated with other diseases in $52.3 \%$ of cases. The family doctor is the specialist consulted before diagnosis in $92.1 \%$ of cases. A total of $50.5 \%$ had a history of psychiatric disorders, these were still present at the time of interview in $36.4 \%$. Around $71 \%$ of the sample received family support, and $70.1 \%$ of cases considered the disease to seriously affect their environment.

Conclusions: Fibromyalgia was mainly diagnosed in women. Subjects with this syndrome have a poor perception of their own health and work situation, and it negatively affects their family environment.

Key words: Fibromyalgia. Psychosocial factors. Health status. Health care system.

(*) Financiación: Red de Centros de Epidemiología y Salud Pública (C03/09) y Red Temática de Investigación de Salud y Género (G03/042) 


\section{INTRODUCCIÓN}

La fibromialgia (FM) es una forma de reumatismo no articular caracterizado por dolor musculoesquelético difuso y crónico, junto a la presencia de múltiples puntos dolorosos a la presión ${ }^{1}$. Los criterios diagnósticos de la FM no fueron definidos hasta 1990 por el American College of Rheumatology (ACR $)^{2}$ $\mathrm{y}$ no fue oficialmente reconocida como enfermedad por la Organización Mundial de la Salud hasta $1992^{3}$. En nuestro país, la prevalencia estimada es de $2,4 \%$ en la población adulta, con un ratio mujer/hombre de $20 / 1(4,2 \% / 0,2 \%)^{4,5}$.

A pesar del gran desconocimiento de la fisiopatogenia de la FM, la teoría más aceptada es que se trata de un trastorno de la modulación del dolor que ocasiona una disminución del dintel de percepción nociceptivo y por tanto hipersensibilidad dolorosa ${ }^{6}$. No existe acuerdo, sin embargo, en el papel que juega el componente psicológico en la FM. Así, mientras algunos autores creen que es similar al que se observa en otras personas con dolor crónico, otros consideran que se trata de una patología esencialmente psiquiátrica. No obstante, esta apreciación es rechazada contundentemente por las afectadas y los afectados, que consideran el malestar psíquico secundario a sus dolencias físicas y reivindican su derecho a que el síndrome sea considerado una enfermedad físi$\mathrm{ca}^{7,8}$.

Dada la cronicidad de la sintomatología de la FM y la incapacidad que a menudo produce el síndrome, éste se asocia a unos altos costes indirectos derivados del absentismo laboral ${ }^{4,9}$, así como directos para los servicios sanitarios $^{9-11}$. Un estudio llevado a cabo recientemente en Holanda puso de manifiesto que la FM representa el 10-20\% de las personas atendidas por primera vez en una consulta de reumatología, lo que supone un $2,1-5,7 \%$ de la actividad a nivel primario de salud y un 5-8\% en el ámbito hospitalario ${ }^{12}$.
Dada la complejidad clínica de estas y estos pacientes y el desconocimiento de su etiopatogenia, la FM supone un reto para el profesional sanitario desde el punto de vista terapéutico ${ }^{13}$. Los resultados de algunos estudios parecen sugerir que la opción más efectiva para paliar los síntomas asociados a este síndrome es la combinación de tratamientos farmacológicos con terapias alternativas ${ }^{14}$. Se ha demostrado que, del extenso arsenal terapéutico, los antidepresivos clásicos, inhibidores de la recaptación de serotonina y de noradrenalina, son los más efica$\operatorname{ces}^{15-16}$. Entre las terapias no farmacológicas más utilizadas se encuentran la psicoterapia, fisioterapia, quiromasajes, acupuntura y ejercicio aeróbico ${ }^{17,18}$.

El gran desconocimiento sobre la FM genera incomprensión en el entorno familiar, social e incluso en el sanitario. Todo ello provoca disminución de la autoestima, frustración y ansiedad en la persona afectada al ver disminuida su calidad de vida, y repercutiendo todo esto en su entorno más cercano $^{19}$. Esta incertidumbre ha originado en nuestro país un gran movimiento de presión por parte de asociaciones de pacientes y familiares, llegando a hacerse eco en los medios de comunicación. Sin embargo, y contradictoriamente a esta repercusión mediática, es muy escasa la información que se tiene sobre el perfil clínico de estas y estos pacientes y la respuesta que obtienen del sistema sanitario. Sólo un estudio en nuestro país aborda el impacto laboral de esta patología ${ }^{9}$, y tres los aspectos más clíni$\cos ^{1,4,9}$

Ante esta ausencia de información, se planteó realizar un estudio con 3 objetivos: (1) conocer el perfil sociodemográfico, clínico y psicosocial de las personas que han recibido diagnóstico de FM; (2) describir la respuesta que obtienen del sistema sanitario, y (3) estudiar la repercusión que este cuadro tiene en sus actividades habituales, incluyendo el ámbito laboral.

Rev Esp Salud Pública 2005, Vol. 79, N. ${ }^{\circ} 6$ 


\section{SUJETOS Y MÉTODOS}

Sujetos: Se realizó un estudio descriptivo transversal mediante encuestas telefónicas durante los meses de junio, julio y agosto de 2004. La población diana estaba constituida por todas las personas diagnosticadas de FM según los criterios del ACR, en tres consultas del Servicio de Reumatología del Hospital Universitario Virgen de las Nieves de Granada en el año $2003(n=258)$. No se incluyeron en el estudio las personas con incapacidad física o psíquica limitante para responder a un cuestionario telefónico $(\mathrm{n}=7)$, ni aquellas que tenían un teléfono incorrecto $(\mathrm{n}=14)$. Las personas no localizadas tras tres llamadas efectuadas en distintos días y franjas horarias ( $\mathrm{n}=19)$, así como aquellas que no quisieron participar $(\mathrm{n}=4)$, fueron considerados pérdidas del estudio. Finalmente se obtuvo información de 214 pacientes, con una tasa de respuesta de 90,3\%. A todos y todas las pacientes que aceptaron participar en el estudio se les garantizó verbalmente la confidencialidad de los datos.

Variables estudiadas e instrumentos de medición: El instrumento utilizado para recoger la información fue un cuestionario expresamente diseñado para este estudio (ver Anexo), que consta de 27 preguntas, 8 abiertas y 19 cerradas o semicerradas. La selección de las variables incluidas en el cuestionario se hizo tras una revisión exhaustiva de otros trabajos sobre el mismo tema ${ }^{9-11}$. Las preguntas pretendían ser claras y concisas para aumentar la fiabilidad. Previamente se realizó un estudio de pilotaje con varios sujetos diagnosticados de FM en el año $2002(n=15)$. De esta forma se identificaron aspectos ambiguos y alguna pregunta de difícil comprensión, además de servir de entrenamiento a las encuestadoras. Además de las variables sociodemográficas habituales (sexo, edad, vivienda habitual, estado civil, número de hijos, nivel educativo, situación laboral, nivel de ingresos), en este estudio se contemplaron las siguientes variables:
Características clínicas: síntomas iniciales y actuales de la FM, síntomas que más afectan al desarrollo de su vida diaria, enfermedades asociadas y salud autopercibida (buena, regular, mala, muy mala).

Características psicosociales: antecedentes familiares y/o personales de patología mental, patología mental actual (considerando por patología mental cualquier tipo de trastorno mental como depresión, ansiedad, somatización, fobias, entre otras), ocurrencia de acontecimientos traumáticos en la infancia y/o a lo largo de su vida (como por ejemplo haber sido víctima de alguna agresión, haber vivido de forma traumática la muerte de algún familiar u otra persona cercana, etc), presencia de apoyo social y repercusiones del síndrome en el entorno familiar.

Características sanitarias: primer especialista consultado y tipo de especialista que deriva a reumatología, tiempo transcurrido desde el inicio de los síntomas hasta el diagnóstico de la FM, número y tipo de especialistas consultados antes y después del diagnostico, tratamiento farmacológico actual para la FM y tratamiento no farmacológico.

Características laborales: situación laboral actual (amas de casa, trabajo remunerado, jubilados y jubiladas, paro laboral y baja laboral), tipo de trabajo actual en personas con trabajo remunerado (manual, no manual), tipo de trabajo a lo largo de la vida (manual, no manual), tipo de baja laboral en pacientes en esta situación (incapacidad temporal o permanente), tipo de trabajo realizado antes de la baja laboral (manual, no manual) y motivo de la baja laboral.

Análisis estadístico: Se realizó un análisis descriptivo, usando frecuencias y porcentajes para las variables cualitativas, y media y desviación típica para las cuantitativas. El análisis de datos se llevó a cabo mediante el programa estadístico SPSS, versión 11.0. Algunas de las variables fueron recodificadas para el análisis. 


\section{RESULTADOS}

La edad media de la muestra fue de casi 47 años, siendo hombres sólo el 7,9\%. El 80,8\% estaban casados y casadas, tenían una media de 2 hijos y el 64,5\% residía en un ámbito rural. Trabajaba en el momento de la entrevista el $60,7 \%$ de la muestra y el $47,7 \%$ tenía un nivel de ingresos mensuales familiares inferiores a $900 €$. El 55,6\% tenía un nivel educativo medio, con estudios primarios y secundarios, y sólo un $15 \%$ tenía estudios superiores.

El síntoma inicial más frecuente es el dolor osteoarticular, en un $67,8 \%$ de los casos. Los síntomas actuales más predominantes en el estudio fueron los reumatológi$\cos (100 \%)$, neurológicos $(97,2 \%)$ y psico- lógicos $(89,7 \%)$. De ellos, el dolor $(66,4 \%)$ es el síntoma que más afecta al desarrollo de la vida diaria, seguido del cansancio $(29,9 \%)$ y el estado de ánimo $(3,7 \%)$. La FM se asocia con otras enfermedades en un 52,3\%, destacando las endocrinas $(25,9 \%)$, cardiovasculares $(18,8 \%)$, reumatológicas $(15,2 \%)$ y ginecológicas $(15,2 \%)$. Finalmente, la población del estudio percibe su salud como mala o muy mala en un $64 \%$ de los casos, regular en un $29 \%$ y buena sólo en un $7 \%$ (tabla 1).

La tabla 2 muestra las características psicosociales de la población estudiada. El $20,1 \%$ de las y los pacientes del estudio tenía antecedentes familiares de patología mental. El 50,5\% había presentado algún trastorno psiquiátrico a lo largo de su vida pero sólo el

Tabla 1

Características clínicas de la muestra $(\mathrm{N}=\mathbf{2 1 4})$

\begin{tabular}{|c|c|c|c|}
\hline & & $\mathbf{N}$ & $\%$ \\
\hline Síntoma inicial & $\begin{array}{l}\text { Dolor osteoarticular } \\
\text { Cansancio } \\
\text { Parestesias }\end{array}$ & $\begin{array}{c}145 \\
57 \\
12\end{array}$ & $\begin{array}{r}67,8 \\
26,6 \\
5,6\end{array}$ \\
\hline Síntomas actuales & $\begin{array}{l}\text { Reumatológicos } \\
\text { Neurológicos } \\
\text { Psicológicos } \\
\text { Circulatorios } \\
\text { Asociados }\end{array}$ & $\begin{array}{l}214 \\
208 \\
192 \\
118 \\
156\end{array}$ & $\begin{array}{r}100,0 \\
97,2 \\
89,7 \\
55,1 \\
72,9\end{array}$ \\
\hline Síntoma que más afecta en su vida diaria & $\begin{array}{l}\text { Dolor osteoarticular } \\
\text { Cansancio } \\
\text { Bajo estado de ánimo }\end{array}$ & $\begin{array}{c}142 \\
64 \\
8\end{array}$ & $\begin{array}{c}66,4 \\
29,9 \\
3,7\end{array}$ \\
\hline \multicolumn{2}{|c|}{$\mathrm{N}^{\circ}$ de pacientes que presentan enfermedades asociadas } & 112 & 52,3 \\
\hline Tipo de enfermedades asociadas & $\begin{array}{l}\text { Endocrinas } \\
\text { Cardiovasculares } \\
\text { Ginecológicas } \\
\text { Reumatológicas } \\
\text { Digestivas } \\
\text { Urológicas/nefrológicas }\end{array}$ & $\begin{array}{l}29 \\
21 \\
17 \\
17 \\
15 \\
13\end{array}$ & $\begin{array}{l}25,9 \\
18,8 \\
15,2 \\
15,2 \\
13,4 \\
11,6\end{array}$ \\
\hline Salud autopercibida & $\begin{array}{l}\text { Buena } \\
\text { Regular } \\
\text { Mala } \\
\text { Muy mala }\end{array}$ & $\begin{array}{l}15 \\
62 \\
82 \\
55\end{array}$ & $\begin{array}{r}7,0 \\
29,0 \\
38,3 \\
25,7\end{array}$ \\
\hline
\end{tabular}


Tabla 2

Características psicosociales de las personas de la muestra $(\mathrm{N}=\mathbf{2 1 4})$

\begin{tabular}{|c|c|c|c|}
\hline \multirow{2}{*}{\multicolumn{2}{|c|}{ Antecedentes familiares de patología mental }} & $\mathbf{N}$ & $\%$ \\
\hline & Antecedentes familiares de patología mental & 43 & 20,1 \\
\hline \multicolumn{2}{|c|}{ Antecedentes personales de patología mental } & 108 & 50,5 \\
\hline \multicolumn{2}{|c|}{$\mathrm{N}^{\circ}$ pacientes con patología mental actual } & 78 & 36,4 \\
\hline \multicolumn{2}{|c|}{$\mathrm{N}^{\circ}$ pacientes que han sufrido acontecimientos traumáticos } & 125 & 58,4 \\
\hline Tipo de acontecimiento traumático & $\begin{array}{l}\text { Trauma infantil } \\
\text { Trauma adulto } \\
\text { No contesta }\end{array}$ & $\begin{array}{l}53 \\
52 \\
20\end{array}$ & $\begin{array}{l}42,4 \\
41,6 \\
16,0\end{array}$ \\
\hline \multicolumn{2}{|c|}{$\mathrm{N}^{\mathrm{o}}$ pacientes que disponen de apoyo social } & 152 & 71,0 \\
\hline Fuente del apoyo social & $\begin{array}{l}\text { Pareja } \\
\text { Familiares } \\
\text { Amigos y amigas }\end{array}$ & $\begin{array}{l}79 \\
56 \\
17\end{array}$ & $\begin{array}{l}52,0 \\
36,8 \\
11,2\end{array}$ \\
\hline \multicolumn{2}{|c|}{$\mathrm{N}^{\circ}$ pacientes a los que la FM causa repercusiones en el entorno familiar } & 150 & 70,1 \\
\hline Tipo de repercusión & $\begin{array}{l}\text { Dependencia de la familia } \\
\text { Disminución vida social } \\
\text { Problemas con la pareja } \\
\text { Sobrecarga familiar }\end{array}$ & $\begin{array}{l}59 \\
37 \\
12 \\
42 \\
\end{array}$ & $\begin{array}{c}39,3 \\
24,7 \\
8,0 \\
28,0\end{array}$ \\
\hline
\end{tabular}

$36,4 \%$ tenía diagnosticada alguna patología mental en el momento de la encuesta. El $69,4 \%$ de las personas con antecedentes de patología mental presentaban una patología mental actual. Sólo un $2,8 \%$ de las personas sin antecedentes referían patología en la entrevista. El 58,4\% de las y los pacientes del estudio habían sufrido algún tipo de acontecimiento traumático a lo largo de su vida, presentando porcentajes similares los traumas en la infancia y en la edad adulta. En cuanto al apoyo social, el $71 \%$ de la muestra refirió tener apoyo, principalmente de la pareja (52\%). La FM repercute en el entorno familiar del 70,1\% de las y los pacientes, provocando su dependencia de los familiares en el 39,3\% de estos casos, agotamiento y sobrecarga en los familiares (28\%), disminución de la vida social $(24,7 \%)$ y problemas con la pareja (8\%).

En cuanto a las características sanitarias, el médico de familia es el especialista más consultado por las personas con FM al inicio de los síntomas $(92,1 \%)$, siendo también este facultativo el que más les deriva a reu- matología (62.6\%). El tiempo transcurrido desde el inicio de los síntomas hasta el diagnóstico de la FM es, como media, de 9,2 años. La media de especialistas consultados antes del diagnóstico es 4. Tras el mismo, sólo consultó un 36,9\% y la media de especialistas fue uno. Antes del diagnóstico las especialidades más consultadas son Traumatología y Rehabilitación (97,2\%), Salud Mental $(62,1 \%)$ y Neurólogía $(39,7 \%)$. Tras el diagnóstico, los pacientes que consultan demandan otro tipo de especialidades, entre ellas Reumatología y Unidad del dolor (tabla 3).

En el momento del estudio consumía algún tratamiento farmacológico el 79,9\% de la muestra. Todos y todas las pacientes tomaban analgésicos y/o AINES, bien solos $(11,1 \%)$ o asociados a otros fármacos, principalmente antidepresivos $(49,1 \%)$ o relajantes musculares y/o ansiolíticos $(24,6 \%)$. Además de los tratamientos farmacológicos, realizaban tratamientos no farmacológicos el $45,3 \%$ de la muestra, siendo el ejercicio físico el más frecuente $(51,5 \%)$ (tabla 3$)$. 
Tabla 3

Características Sanitarias de las personas de la muestra $(\mathrm{N}=\mathbf{2 1 4})$

\begin{tabular}{|c|c|c|c|}
\hline & & $\mathbf{N}$ & $\%$ \\
\hline \multirow[t]{2}{*}{ Primer/a especialista consultado/a } & Médico/a de familia & 197 & 92,1 \\
\hline & Otro/a especialista & 17 & 7,9 \\
\hline \multirow[t]{6}{*}{ Médico/a que deriva al reumatólogo/a } & Médico/a de familia & 134 & 62,6 \\
\hline & Médico/a de empresa & 3 & 1,4 \\
\hline & Asociación fibromialgia & 1 & 0,5 \\
\hline & Especialista médico hospitalario & 23 & 10,7 \\
\hline & Especialista quirúrgico hospitalario & 45 & 21,0 \\
\hline & Contacto directo & 8 & 3,7 \\
\hline \multirow[t]{5}{*}{$\begin{array}{l}\text { Especialistas consultados/as antes del } \\
\text { diagnóstico }\end{array}$} & Traumatólogo/a o rehabilitador/a & 208 & 97,2 \\
\hline & Salud mental & 133 & 62,1 \\
\hline & Neurólogo/a & 85 & 39,7 \\
\hline & Digestivo/a & 48 & 22,4 \\
\hline & Endocrino/a & 13 & 6,1 \\
\hline \multicolumn{2}{|l|}{ Consulta médica tras el diagnóstico } & 79 & 36,9 \\
\hline \multirow{6}{*}{$\begin{array}{l}\text { Tipo especialistas consultados/as después del } \\
\text { diagnóstico }\end{array}$} & Reumatólogo/a & 21 & 26,6 \\
\hline & Traumatólogo/a o rehabilitador/a & 14 & 17,7 \\
\hline & Unidad del dolor & 12 & 15,2 \\
\hline & Salud mental & 11 & 13,9 \\
\hline & Digestivo/a & 9 & 11,4 \\
\hline & Otros/as & 12 & 15,2 \\
\hline \multicolumn{2}{|c|}{ 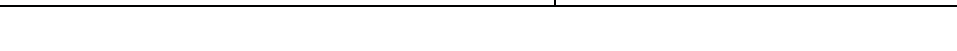 } & media & DE \\
\hline \multicolumn{2}{|c|}{ Tiempo trascurrido hasta el diagnóstico de FM (años) } & 9,2 & 7,1 \\
\hline \multicolumn{2}{|c|}{$\mathrm{N}^{\circ}$ especialistas consultados/as antes del diagnóstico } & 4,0 & 1,7 \\
\hline \multirow{2}{*}{\multicolumn{2}{|c|}{$\mathrm{N}^{\circ}$ especialistas consultados/as después del diagnóstico }} & 1,2 & 0,5 \\
\hline & & $\mathbf{N}$ & $\%$ \\
\hline \multicolumn{2}{|l|}{$\mathrm{N}^{\circ}$ pacientes con tratamiento farmacológico actual } & 171 & 79,9 \\
\hline \multirow[t]{5}{*}{ Tipo de tratamiento farmacológico } & Analgésicos/AINES solos & 19 & 11,1 \\
\hline & + Antidepresivos & 84 & 49,1 \\
\hline & +RM/ansiolíticos & 42 & 24,6 \\
\hline & $+\mathrm{GABA}$ & 18 & 10,5 \\
\hline & +Reguladores del cartílago/Calcio & 8 & 4,7 \\
\hline \multicolumn{2}{|c|}{$\mathrm{N}^{\circ}$ pacientes con tratamiento no farmacológico actual } & 97 & 45,3 \\
\hline \multirow[t]{5}{*}{ Tipo de tratamiento no farmacológico } & Ejercicio físico & 50 & 51,5 \\
\hline & Fisioterapia/Rehabilitación & 26 & 26,8 \\
\hline & Medicina alternativa & 6 & 6,2 \\
\hline & Ozonoterapia & 7 & 7,2 \\
\hline & Técnicas de relajación & & 8,2 \\
\hline
\end{tabular}


Tabla 4

Características laborales de la muestra $(\mathrm{N}=\mathbf{2 1 4})$

\begin{tabular}{|l|l|c|c|}
\hline \multicolumn{2}{|c|}{} & $\mathbf{N}$ & $\%$ \\
\hline Situación laboral actual & Pacientes activos: & & \\
& $\quad$ Amas de casa & 81 & 37,8 \\
& Trabajo remunerado & 49 & 22,9 \\
& Pacientes inactivos: & & \\
& Jubilados/as & 5 & 5,6 \\
& Paro laboral & 12 & 2,3 \\
& Baja laboral & 67 & 31,4 \\
& & & \\
\hline Tipo de la baja laboral (N=67) & Incapacidad Temporal & 35 & 52,2 \\
& Incapacidad Permanente & 32 & 47,8 \\
\hline Motivo de la baja laboral & Dolor osteoarticular & 50 & 74,6 \\
& Cirugía Traumatológica/Plástica & 6 & 9,0 \\
& FM & 11 & 16,4 \\
\hline Tipo de trabajo antes de la baja (n=67) & Trabajo manual & 43 & 64,1 \\
& Trabajo no manual & 24 & 35,9 \\
\hline Tipo de trabajo actual en pacientes activos & Trabajo manual & 28 & 57,1 \\
(n=49) & Trabajo no manual & 21 & 42,9 \\
\hline Tipo de trabajo a lo largo de la vida (n=142) & Trabajo manual & 127 & 89,4 \\
& Trabajo no manual & 15 & 10,6 \\
\hline
\end{tabular}

La tabla 4 muestra las características laborales de los y las pacientes. El 39,3\% de la muestra incluida en el estudio no trabajaba en el momento de la entrevista, bien porque estaban jubilados o jubiladas $(5,6 \%)$, en paro laboral $(2,3 \%)$ o con una baja laboral $(31,4 \%)$. El tipo de incapacidad por la que estas personas están de baja es temporal en el $52,2 \%$ de los casos. El motivo que justifica estas bajas laborales es mayoritariamente el dolor osteoarticular (74,6\%), destacando en un $16,4 \%$ el diagnóstico de fibromialgia y sólo en un $9 \%$ la cirugía traumatológica y/o plástica. Antes de la baja laboral, el 64,8\% de la muestra refiere haber desempeñado un trabajo de tipo manual. De las personas que trabajan, el 37,8\% son mujeres amas de casa y el $22,9 \%$ tienen un trabajo remunerado. De éstos, el $57,1 \%$ realiza trabajos manuales y el 42,9\% no manuales. A lo largo de la vida, el $89,4 \%$ desempeñó trabajos de tipo manual.

\section{DISCUSIÓN}

La FM causa baja laboral en un gran porcentaje de casos y casi dos tercios de los pacientes perciben su salud como mala o muy mala. La mayoría recibe tratamiento farmacológico y casi la mitad se somete a tratamientos no farmacológicos. La mitad de la muestra refiere tener antecedentes de patología mental y la mayoría destaca un fuerte impacto del síndrome en su entorno familiar. Merece la pena mencionar que el tiempo medio transcurrido desde el inicio de los síntomas hasta el diagnóstico del síndrome es de 9 años.

Teniendo en cuenta, por una parte, la escasez de estudios sobre FM en nuestro país y, por otra, las enormes repercusiones del síndrome tanto en la calidad de vida de los y las pacientes como en el gasto sanitario, el estudio del perfil sociodemográfico, clínico y 
psicosocial de estas personas, la repercusión de la FM en su vida laboral y la respuesta del sistema sanitario resulta imprescindible como primer paso para conocer la realidad de este síndrome.

Antes de la discusión de los resultados obtenidos deben señalarse algunas posibles limitaciones del estudio derivadas principalmente de la patología estudiada, concretamente de la posibilidad de sobre o infradiagnóstico. Esto viene determinado por la formación del especialista y su convicción personal en cuanto a la consideración de la FM como una verdadera entidad clínica. También es importante considerar la sensibilidad y la especificidad de los actuales criterios diagnósticos de la misma, cuestionados por algún autor ${ }^{20}$. El sesgo por pérdidas (pacientes no localizados y que no quisieron responder al cuestionario) no es relevante, dada la elevada tasa de respuesta.

Las características sociodemográficas y clínicas de las personas estudiadas fueron similares a las descritas en estudios anteriores $^{1,4,9}$. Igualmente, datos semejantes a los obtenidos en este estudio se observaron en otros trabajos previos, en relación al tiempo transcurrido hasta el diagnóstico de FM, el número y tipo de especialistas consultados antes del mismo ${ }^{4}$. El hecho de que tras el diagnóstico se consulte como media un solo especialista no es muy valorable, teniendo en cuenta que las personas fueron diagnosticadas en el año 2003. En cuanto al tratamiento, hay que decir que en el momento en que se realizaron las entrevistas casi el $80 \%$ tomaban algún tipo de fármaco, fundamentalmente la combinación de analgésicos/ AINES con antidepresivos, que se ha demostrado como la más efectiva dentro del arsenal terapéutico farmacológico ${ }^{21}$. Según lo recogido en recientes investigaciones, los tratamientos no farmacológicos son muy demandados y efectuados por las y los pacientes con FM. Una revisión sistemática recogida en la Cochrane Library concluye que la educación combinada con el entrena- miento físico produce efectos positivos en pacientes con $\mathrm{FM}^{22}$. En este estudio, sin embargo, aquellas personas que realizan alguna terapia no farmacológica no superan el $50 \%$. De éstas, los tipos más frecuentes son el ejercicio físico, la fisioterapia y la rehabilitación, probablemente porque las otras alternativas son más caras y de difícil acceso para muchas de las personas encuestadas.

Casi dos tercios de la muestra del estudio perciben su salud como mala o muy mala. Esto contrasta con los datos de la Encuesta Andaluza de Salud realizada en el año 2003, donde la salud fue percibida como muy buena y buena por el $73,6 \%$ de las personas encuestadas $^{23}$. La FM es una patología crónica que, aunque no provoca lesiones en los huesos ni deforma las articulaciones, conlleva una alteración en la capacidad funcional del paciente y de la paciente lo suficientemente importante como para que presente considerables dificultades para el desarrollo de las actividades diarias habituales, mayores incluso que las que pueden tener las personas afectas de artritis reumatoide y artrosis degenerativa ${ }^{12}$.

El estudio del papel de los factores psicosociales en el estado de salud de los y las pacientes con FM ha sido abordado en diversos trabajos ${ }^{24,25}$. En nuestro estudio, el $58,4 \%$ de la muestra había sufrido algún acontecimiento traumático a lo largo de su vida, resultado corroborado por otros estu$\operatorname{dios}^{24}$. Es relevante el elevado apoyo social que tienen estas personas, sobre todo ofrecido por la pareja y familiares, lo que les permite disponer de un recurso importante para enfrentar el aspecto emocional de la FM, a veces más difícil que el físico. Llama la atención que el $70 \%$ de la muestra percibía que el síndrome tenía importantes repercusiones en su vida familiar, sobre todo generando gran dependencia de sus familiares y provocando agotamiento y sobrecarga en los y las que le rodean. 
Finalmente, uno de los temas más debatidos en la FM es su relación con los trastornos psicológicos y psiquiátricos. En este estudio se encontraron antecedentes personales de patología mental en el 50\% de los casos. Sin embargo, en el momento de la entrevista era de $36,4 \%$, datos similares a los obtenidos en trabajos previos y en otras patologías crónicas $^{8}$. Es posible que una vez conocido que presentan una patología llamada Fibromialgia, la ansiedad ante la incertidumbre descienda a niveles de normalidad. Esto era verbalizado por los y las pacientes del estudio como «estoy mejor una vez que sé que no me lo estoy inventado». Por último, sólo el 2,8\% de las personas sin antecedentes de patología mental previa al diagnóstico presentan un trastorno de este tipo tras el mismo, lo que parece contradecir la hipótesis de que la FM pueda ser una entidad puramente psiquiátrica.
Para concluir, merece la pena destacar que, a pesar de la incertidumbre que existe en torno a la FM, sí parece claro el hecho de que habría que intentar evitar la demora en el diagnóstico, tan angustiosa para los y las pacientes y tan costosa para el sistema sanitario. Asimismo, y tras lo percibido en las entrevistas con estas personas, un aspecto muy importante en el planteamiento terapéutico de los y las pacientes con FM sería informarles desde un principio de las diferentes posibilidades terapéuticas de la patología que padecen. La información debería ser directa, objetiva y acorde con el conocimiento científico que se posee sobre la misma. De este modo la persona podría adaptarse y tendría una expectativa adecuada de la patología crónica que padece. Se hace necesario realizar un análisis individualizado de cada paciente, valorando todo el cuadro sintomatológico que presenta mediante un abordaje multidisciplinario ${ }^{26}$. 


\section{ANEXO I \\ Encuesta telefónica}

Caso $\mathrm{N}^{\mathrm{o}}$ :

\section{VARIABLES SOCIODEMOGRÁFICAS}

1.- Nombre y apellidos:

2.- $\mathrm{N}^{\circ}$ de teléfono:

3.- Edad: ....... años.

4.- Sexo: $M / F$

5.-Vivienda habitual:

- Ámbito rural (1) - Ámbito urbano (2)

6.- Estado Civil:

-Soltero/a. (1)

-Casado/a_En pareja (2)

- Divorciado/a_Separado/a (3)

-Viudo/a. (4)

7.- $\mathrm{N}^{\mathrm{O}}$ de hijos:

8.- Nivel educativo:

-No sabe leer ni escribir (1)

-Sin estudios (2)

-Estudios Primarios (3)

-Estudios Secundarios (4)

-Estudios Superiores (5)

9.- Situación Laboral:

-Trabaja en casa (1)

-Asalariado/a._Autónomo/a (2)

-Desempleado/a. (3)

-Jubilado/a ( $>65$ años) (4)

-Baja Laboral (5): Tipo de BL: Incapacidad temporal (A)/ Incapacidad permanente (B)

Razón de la B L:

Tipo de trabajo que realizaba antes de la BL:

9.1.-¿Ha realizado algún otro trabajo a lo largo de su vida?

Sí (1): Tipo:......

No (2)

10.- Nivel de ingresos:

-Menos de 600 Euros/mes. (1)

-De 600 a 900 Euros/mes. (2)

-De 900 a 1200 Euros/mes. (3)

-Más de 1200 Euros/mes. (4)

\section{VARIABLES CLÍNICO-SANITARIA}

11.-¿Cuál/es fueron los primeros síntomas de la enfermedad y cuándo aparecieron (año)?

12.- Actualmente, ¿qué otros síntomas presenta?

\begin{tabular}{|c|c|c|c|}
\hline 1 & Dolor generalizado & 2 & Sueño no reparador \\
\hline 3 & Cansancio/fatiga & 4 & Rigidez matinal \\
\hline 5 & Parestesias y/o Sd. Túnel carpiano & 6 & Cefalea \\
\hline 7 & Pérdida de fuerza & 8 & Problemas circulatorios \\
\hline 9 & Ansiedad o nerviosismo & 10 & Depresión \\
\hline 11 & Sensación de inflamación/tumefacción en articulaciones & 12 & Sindrome de intestino irritable \\
\hline 13 & Vejiga irritable & 14 & Fenómeno de Raynaud \\
\hline 15 & Síndrome seco & 16 & Sindrome premenstrual \\
\hline 17 & Dolor ATM & 18 & Dificultad para concentrarse \\
\hline 19 & Confusión al hablar y/o escribir & 20 & \begin{tabular}{|l|} 
Torpeza en actividades diarias \\
\end{tabular} \\
\hline 21 & Sd. piernas inquietas & 22 & 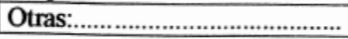 \\
\hline
\end{tabular}

13.- De todos ellos, ¿cuál/es afecta/n más al desarrollo de su vida diaria?: 
14. ¿Cuál fue el primer médico al que consultó por alguno de estos sintomas? Médico de Familia (1). Año:

Otro especialista (2). Tipo y año:

15. ¿ ¿Le prescribió algún medicamento?

Si (1). Tipo:

No (2): No lo creyó oportuno (A)/La paciente ya estaba tomando analgésicos/antiinflamatorios (B) No sabe/no recuerda (3)

16.- ¿A través de qué médico contactó usted con el Reumatólogo?:

17. ¿Cuánto tiempo transcurrió desde que empezó con esta sintomatología reumatológica hasta que le diagnosticaron la FM?: .........................................

18.-¿Con cuántos y a qué tipo de especialistas ha consultado acerca de estos síntomas? Antes del diagnóstico: Después del diagnóstico:

19. - ¿ Está tomando actualmente algún tratamiento farmacológico? Sí (1). Tipo:

No (2)

No sabe/No recuerda (3)

20.- ¿Además del tratamiento farmacológico, realiza algún otro tratamiento alternativo? Sí (1). Tipo

No (2)

21.- ¿Ha sido usted diagnosticada de alguna otra enfermedad?

Sí (1): Tipo:

No (2)

\section{FACTORES PSICOSOCIALES}

22.- ¿Existe algún antecedente familiar de patología mental diagnosticada por un médico?

Sí (1). Tipo:

No (2)

No sabe/no recuerda (3).

23.- ¿Existe algún antecedente personal de patología mental diagnosticada por un médico?

Sí (1)

Antes del diagnóstico de FM. Tipo y año.

Actualmente. Tipo

No (2)

No sabe/no recuerda (3).

24. - ¿Recuerda usted algún acontecimiento traumático en la infancia y/o a lo largo de su vida?

Sí (1). Tipo:

No (2)

25.- ¿Tiene usted a alguna persona/s en la que poder desahogarse cuando tiene problemas?

Si (1). Quién:

No (2)

\section{IMPACTO DE LA ENFERMEDAD EN EL ENTORNO FAMILIAR}

26. - ¿Cree que la enfermedad ha repercutido en la rutina diaria de su familia más cercana?

Sí (1). En qué medida: No (2)

SALUD AUTOPERCIBIDA

27.- ¿Cómo diría usted que es su salud actual?

Muy buena (1) Buena (2) Regular (3) Mala (4) Muy màla (5)

\section{CUESTIONARIO DEL IMPACTO DE LA FIBROMIALGIA (ANEXOI)}




\section{BIBLIOGRAFÍA}

1. Álvarez Lario B, Alonso Valdivieso JL, Alegre López J. Síndrome de fibromialgia: características clínicas de las pacientes españolas. Rev Esp Reumatol. 1996; 23:76-82.

2. Wolfe F, Smythe HA, Yunnus MB, Bennet RM, Bombardier C, Goldenberg DL, et al. The American College of Rheumatology 1990 criteria for the classification of fibromyalgia. Artritis Rhem 1990; 33:160-72.

3. Consensus Document on Fibromyalgia. The Copenhagen Declaration. Journal of Musculosketal Pain, Vol 1. New York: Haworth Press; 1993.

4. Valverde M, Juan A, Rivas B, Carmona L. Fibromialgia. En: Estudio EPISER. Prevalencia e impacto de las enfermedades reumáticas en la población adulta española. Madrid: Msd y Sociedad Española de Reumatología; 2001: p. 77-91.

5. Campos Sánchez S. Fibromialgia. Rev Esp Reumatol. $2000 ; 27: 417-20$.

6. Price DD, Staud R. Neurobiology of fibromyalgia syndrome. J Rheumatol Suppl. 2005; 75:22-8.

7. Martínez E, González O, Crespo JM. Fibromialgia: definición, aspectos clínicos, psicológicos, psiquiátricos y terapéuticos. Salud Mental 2003; 4:27.

8. Okifuji A, Turk DC, Sherman JJ. Evaluation of the relation-ship between depression and fibromyalgia syndrome: why aren't all patients depressed? J Rheumatol. 2000; 27:212-19.

9. Albornoz J, Povedano J, Quijada J, De la Iglesia JL, Fernández A, Pérez-Vilchez D et al. Características clínicas y sociolaborales de la fibromialgia en España: descripción de 193 pacientes. Rev Esp Reumatol 1997; 24:38-44.

10. Boch Romero E, Sáenz Moya N, Valls Esteve M, Víñolas Valer S. Estudio de la calidad de vida en pacientes con fibromialgia: impacto de un programa de educación sanitaria. Aten Primaria 2002; 30:16-21.

11. Tornero J, Vidal J. Impacto social y económico de las enfermedades reumáticas: la discapacidad laboral. Rev Esp Reumatol 1999; 26:357-66.
12. Boonen A, Van Den Heuvel R, Van Tubergen A, et al. Large differences in cost-of-illness and well being between patients with fibromyalgia, chronic low back pain or ankylosing spondylitis. Ann Rheum Dis 2005; 64:396-402.

13. Patkar AA, Biloll, Masand Ps. Management of Fibromyalgia. Curr Psychiatry Rep 2003; 5:21824.

14. Turk DC, Okifuji A, Starz TW, Sinclair JD. Differential responses by psychosocial subgroups of fibromyalgia syndrome patients to an interdisciplinary treatment. Arthritis Care Res 1998; 11:397-404.

15. Arnold LM, Keck PE, Welge JA. Antidepressant treatment of fibromyalgia. A meta-analysis and review. Psychosomatics 2000; 41:104-13.

16. López-Pousa, Lombardía C, Ortega E, Novell R. Eficacia de un fármaco GABAérgico (GamalateB6) sobre la calidad de vida de los enfermos con Fibromialgia. Psiquis 2002; 23:31-38.

17. Taggart HM, Arslanian CL, Bae S, Singh K. Effects of Tai Chi exercise on fibromyalgia symptoms and health-related quality of life. Orthop Nurs 2003; 22:353-60.

18. Ramsay C, Moreland J, Ho M, Joyce S, Walker S, Pullar T. An observer-blinded comparison of supervised and unsupervised aerobic exercise regimens in fibromyalgia. Rheumatology 2000 ; 39:501-5.

19. Wolfe F, Michaud K. Severe rheumatoid arthritis (RA), worse outcomes, comorbidity illness, and sociodemographic disadvantage characterize patients with fibromyalgia. J Rheumatol 2004; 31:696-700.

20. Fitzcharles MA, Esdaile JM. The overdiagnosis of fibromyalgia syndrome. Am J Med. 1997; 103:4450 .

21. Goldenberg DL. Update on the treatment of fibromyalgia. Bulletin on the Rheumatic Diseases 2004; 342:906-9.

22. Karjalainen K, Malmivaara A, van Tulder M, Roine R, Jauhiainen M, Hurri H, Koes B. La rehabilitación multidisciplinaria para la fibromialgia y el dolor musculoesquelético en adultos en edad activa. En: La Cochrane Library plus en español. Oxford: Update Software. 
CARACTERÍSTICAS CLÍNICAS Y PSICOSOCIALES DE PERSONAS CON FIBROMIALGIA. REPERCUSIÓN DEL DIAGNÓSTICO...

23. Consejería de Salud de la Junta de Andalucía. Encuesta Andaluza de Salud. Muestra de Adultos 2003. Sevilla: Consejería de Salud de la Junta de Andalucía; 2003 (datos sin publicar).

24. Walker FA, Keergan D, Gardner D, Sullivan M, Bernstein D, Katon WJ. Psychosocial factors in fibromyalgia compared with rheumatoid arthritis. II. Sexual, physical and emotional abuse, and neglect. Psychosom Med 1997; 59:572-77.
25. Taylor ML, Trotter DR, Csuka ME. The prevalence of sexual abuse in women with fibromyalgia. Arthritis Rheum 1995; 38:9229-34.

26. Collado A, Torres X, Arias A, Cerdà D, Vilarrasa $\mathrm{R}$, Valdés $\mathrm{M}$ et al. Eficacia del tratamiento multidisciplinario del dolor crónico incapacitante del aparato locomotor. Med Clin (Barc) 2001; 117: $401-5$. 